Journal of Engineering and Science Research 2 (2): 38-45, 2018

e-ISSN: 2289-7127

(C) RMP Publications, 2018

DOI: $10.26666 /$ rmp.jesr.2018.2.6

\title{
Development of a Rapid Manufacturing Process for Concave and Convex Lens Arrays
}

\author{
Chih-Yuan Chang \\ Department of Mold and Die Engineering, National Kaohsiung University of Applied Sciences, \\ 415, Chien-Kung Rd., Kaohsiung 807, Taiwan.
}

\begin{abstract}
This paper reports a rapid manufacturing process for the production of concave and convex lens arrays on the polymer substrate. In this method, many small steel balls with highly polished surfaces were placed in a rectangular cavity to form a closely packed small steel ball array. Then, a polymer substrate (PMMA sheet) was placed on top of the small steel ball array, and the stack of the PMMA sheet and the small steel ball array was placed in a hot embossing machine. During the hot embossing process operation, a concave lens array pattern is directly fabricated onto a polymer substrate. In addition, the diameter and depth of the concave lens array can be changed and controlled by adjusting the processing conditions of the hot embossing process. Thus, concave lens arrays with different dimension can be fabricated. Next, the polymer substrate with concave lens array pattern can be used as a mold for rapid replication of polymer convex lens array using vacuum-assisted UV molding process. In this way, various concave and convex lens arrays can be rapid fabricated with high throughput and low cost.
\end{abstract}

Key words: Concave lens array, Convex lens array, Hot embossing, UV molding

\section{INTRODUCTION}

In recent years, polymer lens arrays have been used widely in various optical communication systems, optoelectronic instruments and biomedical systems. Many polymer convex lens arrays and concave lens arrays have also been implemented in advanced light management systems, such as optical fiber systems, image displays, charge-coupled device cameras and light beam-shaping device of laser apparatus. Therefore, the fabrication method for polymer lens arrays is an important issue of concern. Over the years, many methods have been proposed and developed for the fabrication of convex polymer lens arrays on millimeter or micrometer-scales, such as thermal reflow process [1], laser ablation process [2], ink-jet printing process [3], modified LIGA process [4], gray scale photolithography process [5] and polymer replication process [6-9]. Unfortunately, most of these methods are not suitable for direct fabrication of negative or concave lens arrays. In the past, only a few technologies have shown the potential to fabricate concave lens array, such as UV proximity printing technique [10], 3D diffuser lithography [11], combined of thermal reflow technique and PDMS replication process [12], laser-enhanced local wet etching process [13], isotropic etching of glass master [14]. Nevertheless, the preceding literature survey shows that most of the fabrication techniques for concave and convex lens arrays are complex, time consuming and require expensive. Here, unlike previous reports, we propose a simple, low cost and rapid method to fabricate concave and convex lens arrays with different dimension. The method involves a hot embossing process with a polymer substrate and a small steel ball array. Only one embossing step is required, which a concave lens array pattern is directly fabricated onto a polymer substrate. By adjusting the processing conditions of the hot embossing process, the diameter and depth of the concave lens array can be changed and controlled. Thus, concave lens arrays with different dimension can be fabricated. Next, these polymer substrates with different concave lens array patterns can be used as molds for rapid replication of polymer convex lens arrays in the vacuum-assisted UV molding process. In this study, the effects of the hot embossing

Corresponding Author: Chih-Yuan Chang, Department of Mold and Die Engineering, National Kaohsiung University of Applied Sciences, 415, Chien-Kung Rd., Kaohsiung 807, Taiwan. cychang@ kuas.edu.tw 
processing conditions on the diameter and depth of the fabricated concave lens array molds were investigated. After undergoing a vacuum-assisted UV-molding process, the replication quality and optical properties of the polymer convex lens array device were measured and analyzed.

\section{EXPERIMENT}

\section{Experimental Setup}

Figure 1 show the hot embossing machine used in this study. The machine is composed of top and bottom heating plates with a cooling system (The maximum embossing temperature is $200^{\circ} \mathrm{C}$ ), a pneumatic cylinder with a pressure regulator (The maximum embossing force is $2000 \mathrm{~N}$ ), and a power distribution box with an air compressor. The embossing temperature, embossing force and embossing time can be precisely controlled. Figure 2 shows a photograph of a PMMA sheet on a closely packed small steel ball array. In this study, high precise steel balls with highly spherical and polished surfaces are used. The diameter of the small steel balls was $2 \mathrm{~mm} \pm 0.08 \mu \mathrm{m}$. The surface roughness of the small steel balls was $0.01 \mu \mathrm{m}$. This kind of precise steel ball was applied to ball screw industry. Therefore, the accuracy of these steel balls is high and uniform. The dimension layout of the rectangular cavity was $30 \mathrm{~mm} \times$ $30 \mathrm{~mm} \times 3 \mathrm{~mm}$. The dimensions of the PMMA sheets used in this study were $30 \mathrm{~mm} \times 30 \mathrm{~mm} \times 3 \mathrm{~mm}$, and the glass transition temperature of PMMA was approximately $105^{\circ} \mathrm{C}$.

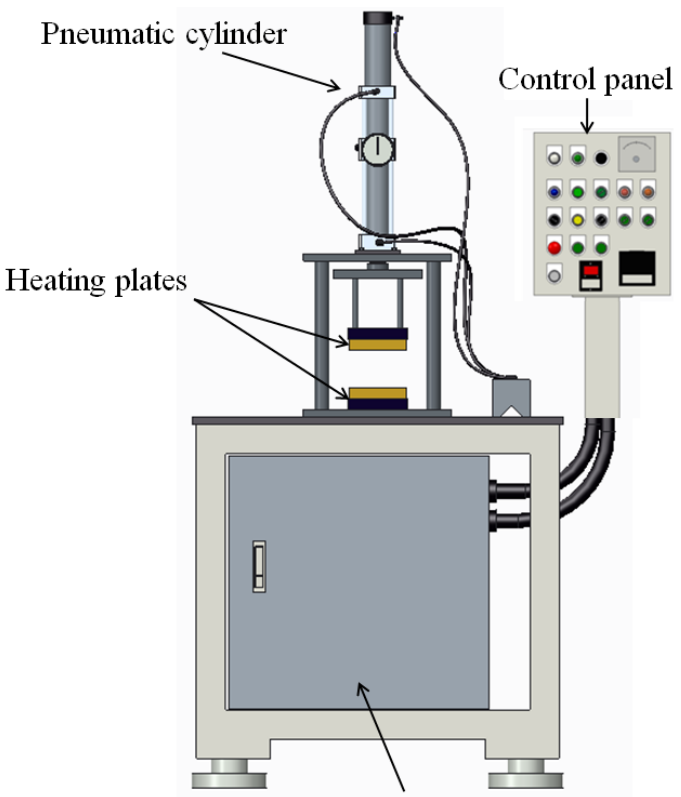

Power distribution box with air compressor

Fig 1 Schematic drawing of the hot embossing machine

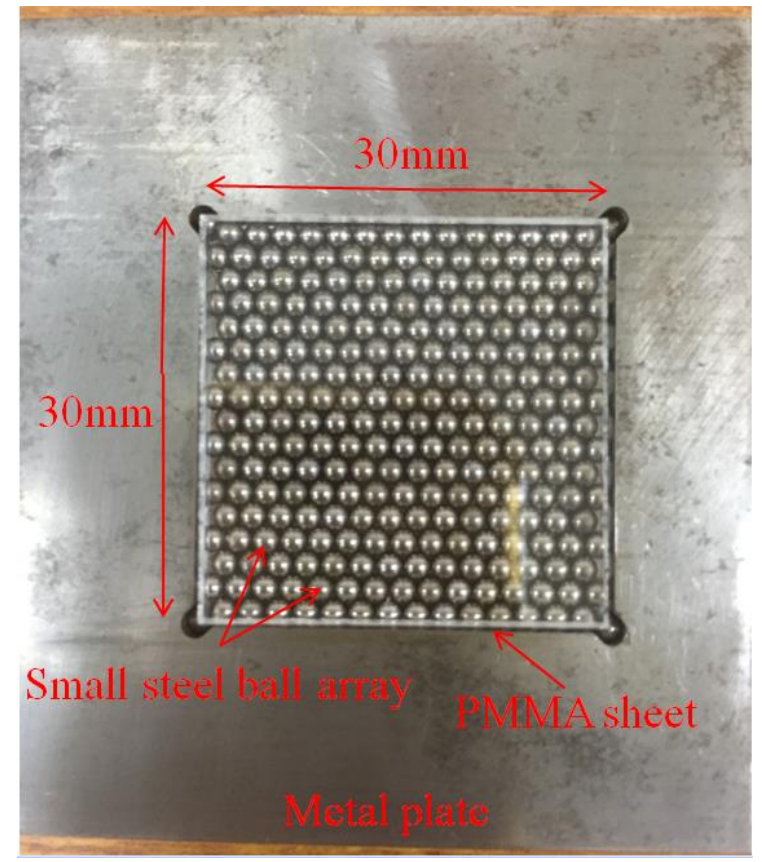

Fig 2 Photograph of a stack of PMMA sheet and small steel ball array

\section{Experimental Method}

The manufacturing process of this research is illustrated in figure 3. First, a rectangular cavity is fabricated on a metal plate using precision milling. Next, a thin layer of Epoxy adhesive was coated in the rectangular cavity. The Epoxy adhesive (Cotronics Corp., USA) offers high bond strength, high temperature stability and low shrinkage. The strength of epoxy adhesives is degraded at temperatures above $250^{\circ} \mathrm{C}$. Then, many extremely precise steel balls with highly spherical and polished surfaces are placed in the cavity to form a closely packed array. When the Epoxy adhesive cured, a closely packed steel ball array was fixed in the rectangular cavity. Then, a PMMA sheet is placed on top of the steel ball array, and the stack of PMMA sheet and steel ball array was put into the hot embossing machine. At this time, the target temperature of heating plates of the hot embossing machine is already reached. Then, top heating plate of the hot embossing machine came down to press the PMMA sheet and the small steel ball array. The softened surface of the PMMA sheet was embossed by the steel ball array under the constant pressure. The concave lens array pattern was formed on the surface of the PMMA sheet due to the plastic visco-elastic deformation principle. When the embossing time period ended, the top heating plate was direct pulled back, and no cooling and packing pressure stage were implemented. Then, the PMMA sheet was de-molded from the steel ball array. A PMMA sheet with a concave lens array pattern was thus obtained. Next, the concave lens array was used as a mold for rapid replication of convex lens array by vacuum-assisted UV molding process. During the vacuum-assisted UV-molding operation, a 
polycarbonate substrate is fixed on a glass plate by adhesive tape. Then, the UV curable polymer layer with thickness of $1.5 \mathrm{~mm}$ is coated on the polycarbonate substrate. A PMMA sheet with concave lens array was placed on the polycarbonate substrate with UV curable polymer layer. Then, the stack of concave lens array, polycarbonate substrate with UV curable polymer layer and glass plate was put into a transparent PET bag. Next, vacuum suction process was operated by a vacuum system with pressure regulator. The vacuum suck pressure is applied to the stack and the concave lens array is filled with UV curable polymer. The UV curable polymer on the polycarbonate substrate is then cured by UV irradiation for 30 seconds. Finally, the vacuum pressure is released and the PMMA concave lens array is removed from the polycarbonate substrate. A UVmolded convex lens array was obtained. The total cycle time of this manufacturing process could be less than 3 minutes.

To study the various processing parameters of the hot embossing process for fabrication of concave lens arrays with various dimensions, three processing parameters, i.e. the embossing temperature, embossing force and embossing time, were chosen. By changing one parameter, with the other parameters fixed at reference states, the effect of each parameter on the diameter and depth of the embossed concave lens array can be determined.

To verify the uniformity of this manufacturing process, the shape and dimension of the fabricated concave and convex lens arrays were measured by a scanning electron microscope (Hitachi S-3000N, Japan) and a surface profiler (Alpha-Step Profilometer, KLATencor). In addition, the replication quality of convex lens arrays was investigated. The replication ratio $(\mathrm{R} \%)$ is defined as $[\mathrm{H} / \mathrm{D}] \times 100 \%$, where $\mathrm{H}$ is the dimension of replicated convex lens arrays and $\mathrm{D}$ is the dimension of the concave lens arrays.

The optical property of the polymer lens arrays were measured by a laser-beam profiling system as shown in figure 4. The laser-beam profiling system were composed of expanding lenses, a mirror, a movable stage with micrometer scale resolution, a CCD camera system with monitor and a He-Ne laser light source. A polymer lens array was fixed on a movable stage, and collimated laser beam illuminates the polymer lens array from the back side. The optical property of the polymer lens array could be measured and determined.

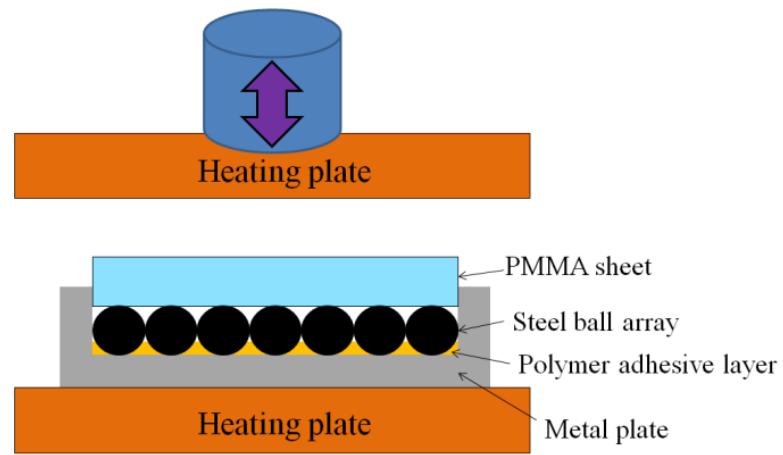

(a) Material preparation

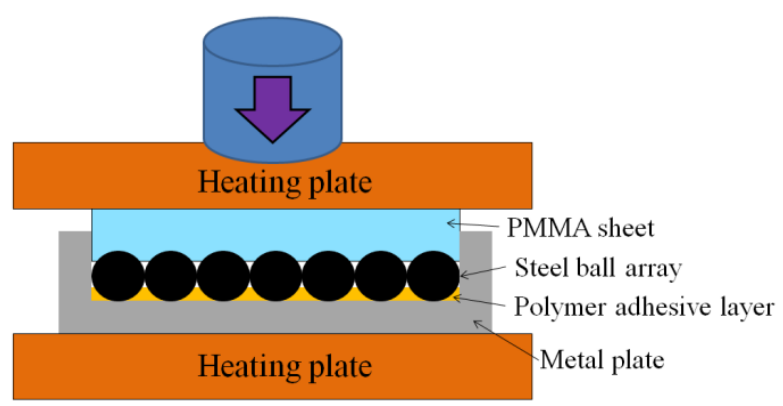

(b) Hot embossing
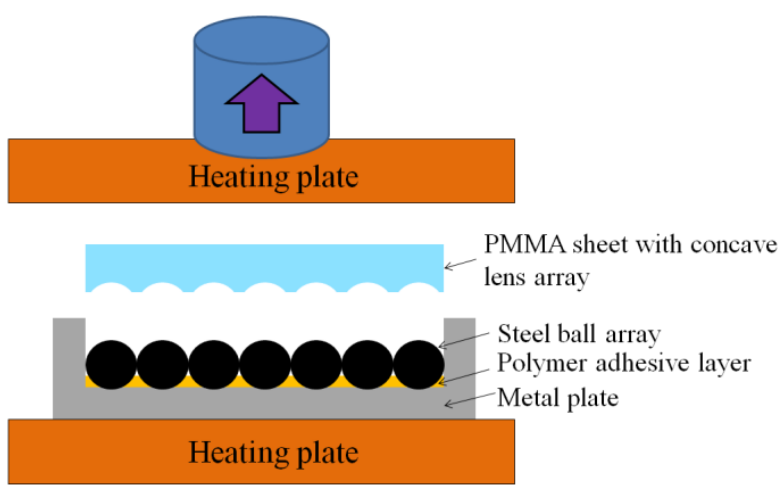

(c) De-molding

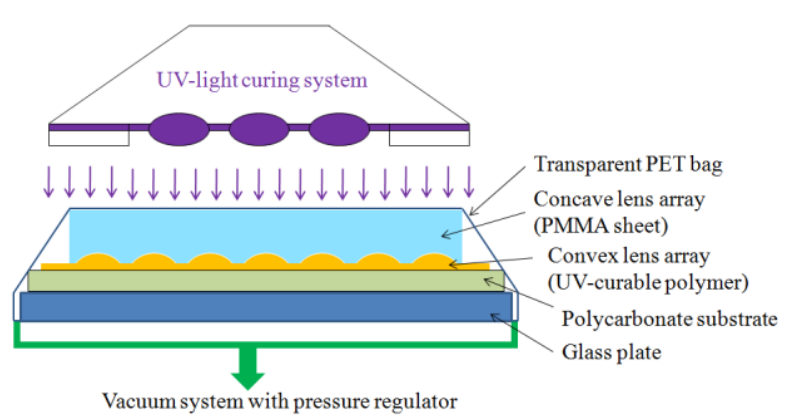

(d) Vacuum-assisted UV-molding process

Fig 3 Manufacturing process of this research 


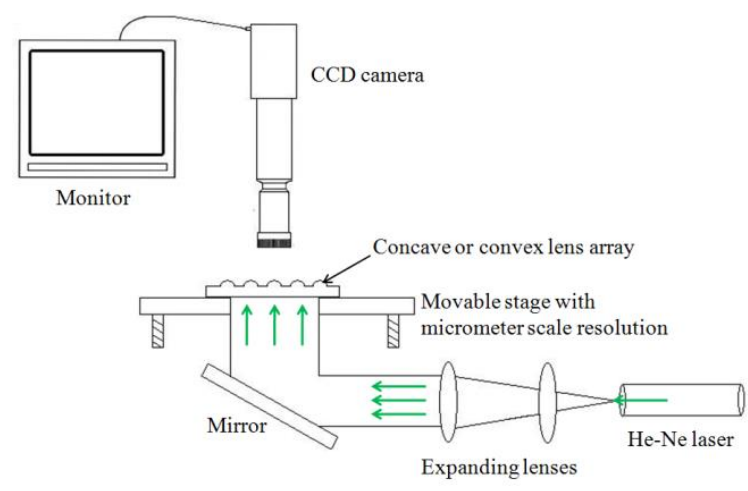

Fig 4 Setup of optical measured system

\section{RESULT AND DISCUSSION}

In the preliminary test, a concave lens array on PMMA sheet was fabricated under the following conditions: embossing temperature $=105^{\circ} \mathrm{C}$, embossing force $=120 \mathrm{~N}$, embossing time $=30 \mathrm{~s}$. A photograph and a SEM image of the fabricated PMMA sheet with concave lens array is shown in figure 5. It is observed that the array of concave lens was successfully fabricated over the whole PMMA sheet. The average diameter of the concave lens array was $550.2 \mu \mathrm{m}$ with a standard deviation of $0.5 \mu \mathrm{m}$. The average depth of the concave lens array was $150.2 \mu \mathrm{m}$ with a standard deviation of $0.4 \mu \mathrm{m}$. The average pitch of the concave lens array was $2002.1 \mu \mathrm{m}$ with a standard deviation of $2.4 \mu \mathrm{m}$. This result indicates good uniformity and controllability of the proposed approach. Figure 6 shows the part of focused light spot pattern and normalized intensity profile from the polymer concave lens array. The images reveal that the pitch and the intensity of the focused light spots are uniform. Next, the surface quality of the polymer concave lens array was measured by atomic force microscope (AFM). The average surface roughness $(\mathrm{Ra})$ of the polymer concave lens array was $12.8 \mathrm{~nm}$, and the minimum surface roughness $(\mathrm{Ra})$ of a single concave lens was $11.7 \mathrm{~nm}$ (Figure 7). These results show that the optical property and surface quality of the polymer concave lens array are good and acceptable for many optoelectronic systems.

To investigate the effect of embossing temperature on the dimension of fabricated concave lens arrays, the embossing temperature was varied from $105^{\circ} \mathrm{C}$ to $135^{\circ} \mathrm{C}$ at a constant embossing force of $200 \mathrm{~N}$ and embossing time at 30 seconds. The result suggests that the average diameter of the embossed concave lens array increase dramatically with the embossing temperature (Figure 8). The diameters of minimum and maximum concave lens array are $550.2 \mu \mathrm{m}$ and $1512.8 \mu \mathrm{m}$, respectively (Figure 9). In addition, the average depth of the embossed concave lens arrays increase from $37.6 \mu \mathrm{m}$ to $264.6 \mu \mathrm{m}$. This result can be explained by that the hardness and viscosity of the PMMA material is decreased with an increase in working temperature, so that a higher volume of the steel ball array is pressed into the PMMA sheet. The average diameter and depth of the fabricated concave lens arrays were thus increased obviously.

Figure 10 presents the effect of embossing force on the dimension of fabricated concave lens arrays. In this experiment, the embossing force was varied from $200 \mathrm{~N}$ to $500 \mathrm{~N}$ at a constant embossing temperature of $105^{\circ} \mathrm{C}$ and embossing time at 30 seconds. The measured result suggests that the average diameter of the embossed concave lens array small increase with the embossing force. In addition, the result also shows that increasing the embossing force results in a very small increase in the depth of fabricated concave lens arrays.

Figure 11 shows the effect of embossing time on the dimension of fabricated concave lens arrays. In this experiment, the embossing time was varied from 30 seconds to 120 seconds while keeping the embossing temperature at $105^{\circ} \mathrm{C}$ and the embossing force at $200 \mathrm{~N}$. The result suggests that increasing the embossing time results in very small change in the diameter and depth of fabricated concave lens arrays. A possible explanation is that the embossing temperature of $105^{\circ} \mathrm{C}$ was set too low for hot embossing of PMMA material. Therefore, the embossing time only has little effect on the dimension of fabricated concave lens arrays.

Based on the above study, the dimension of fabricated concave lens arrays could be changed by adjusting the processing conditions of the hot embossing process. Therefore, various concave lens arrays have been successfully fabricated. In addition, the experimental results imply that the embossing temperature and embossing force plays a major and minor role in the formation of a concave lens array on the PMMA sheet. The embossing time only has little effect on the dimension of fabricated concave lens arrays.

After the PMMA sheets with concave lens array pattern were produced, convex lens arrays were rapid replicated by using vacuum-assisted UV molding process. Figure 12 shows the two types of convex lens arrays produced using PMMA sheets with concave lens array pattern (as seen in figure 9). It is observed that an array of convex lens was successfully replicated. In order to characterize the geometrical uniformity of the convex lens array, the ninety lenses on the polymer substrate are picked and measured. The replication ratio of diameter from a convex lens array is measured as shown in the figure 13 . The replication ratio of diameter was $99.1 \%$ with a standard deviation of $0.9 \%$. The replication ratio of sag height from a convex lens array is measured as shown in the figure 14. The replication ratio of sag height was $98.3 \%$ with a standard deviation of $1.1 \%$. The results suggest that the replication quality of the convex lens produced from the concave lens 
was satisfactory. In addition, the average surface roughness $(\mathrm{Ra})$ of the convex lens array was $13.1 \mathrm{~nm}$.

To demonstrate the optical applications of the convex lens array for the imaging, projection experiment was performed with a mask of " $\mathrm{H}$ " symbol and CCD camera system. The lens array was placed in the middle of the mask and the CCD camera. Then, the miniature image of the focal plane was captured by the CCD camera. Figure 15 shows the clear and non distortion image on the convex lens array. The result show that the convex lens array proved to a good optical imaging performance.

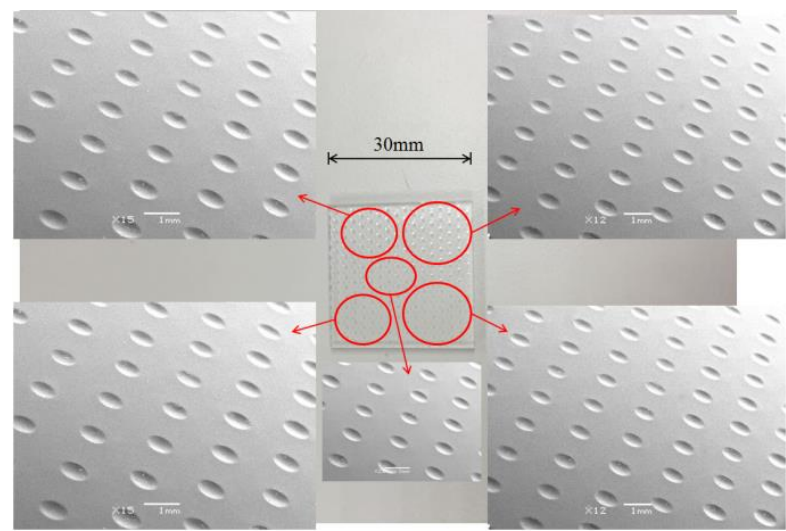

Fig 5 Photograph and SEM images of an embossed concave lens array

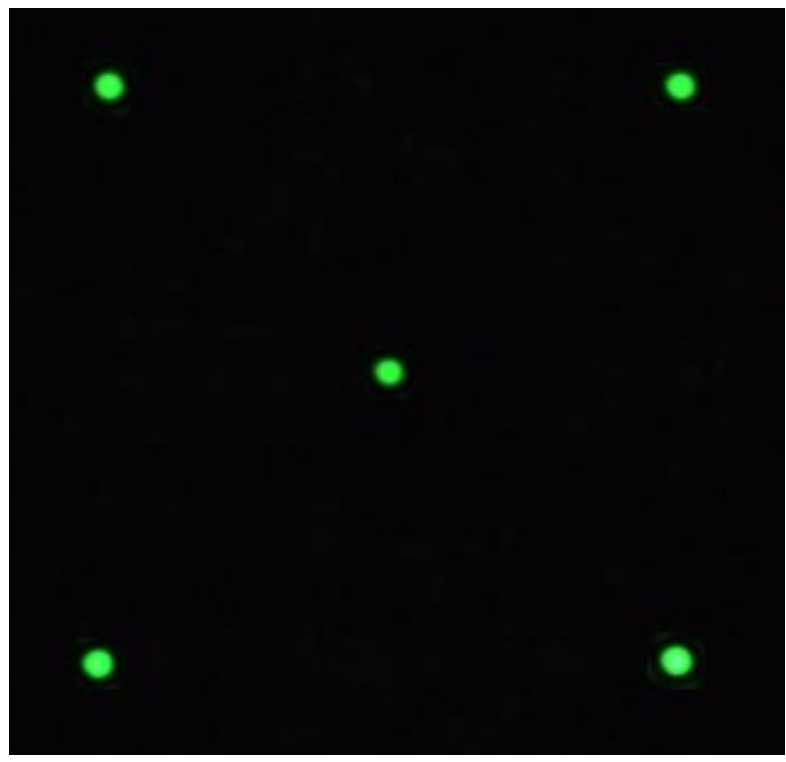

(a) Part of focused light spot pattern of a concave lens array

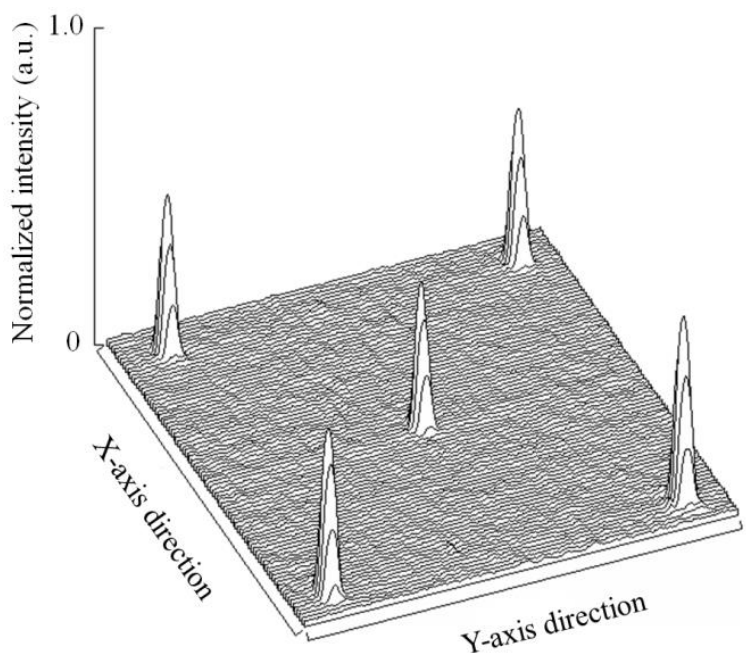

(b) Normalized intensity profile of a concave lens array Fig 6 Laser focused light spot image and normalized intensity profile of a concave lens array

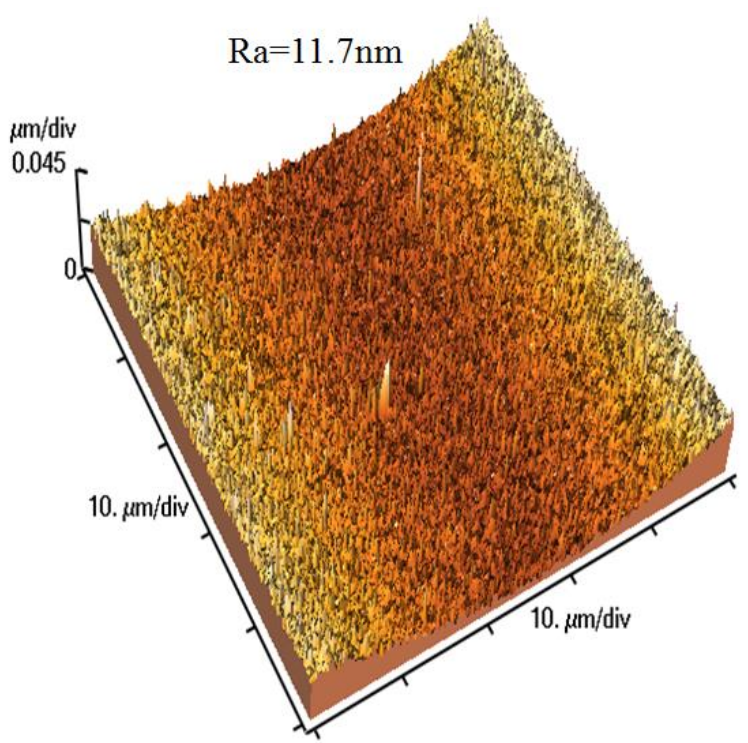

Fig 7 AFM scanning image of a single concave lens

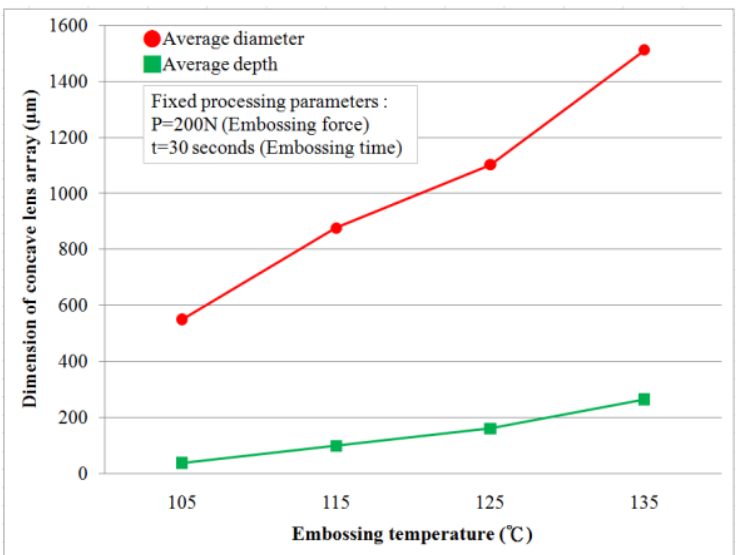

Fig 8 Effect of embossing temperature on the dimension of concave lens arrays 


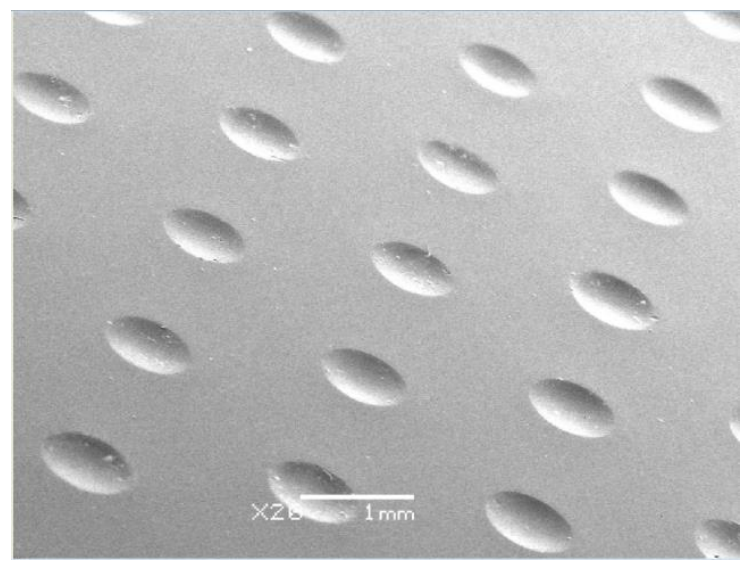

(a) Fabricated concave lens array with diameter of $550.2 \mu \mathrm{m}$

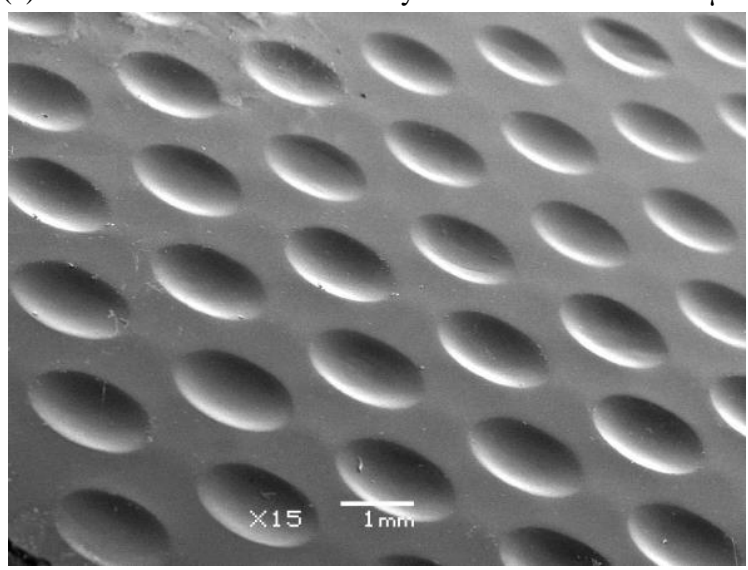

(b) Fabricated concave lens array with diameter of $1512.8 \mu \mathrm{m}$ Figure 9 Fabricated concave lens arrays with different diameter

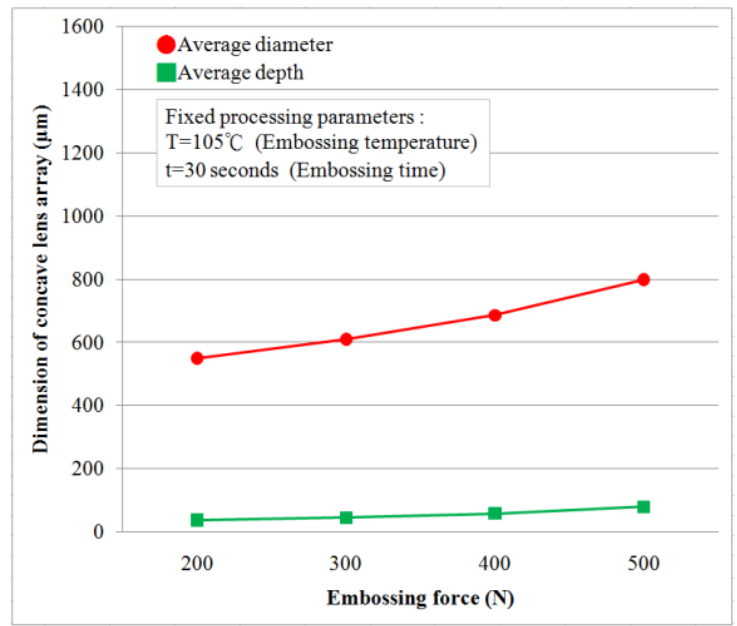

Figure 10 Effect of embossing force on the dimension of concave lens arrays

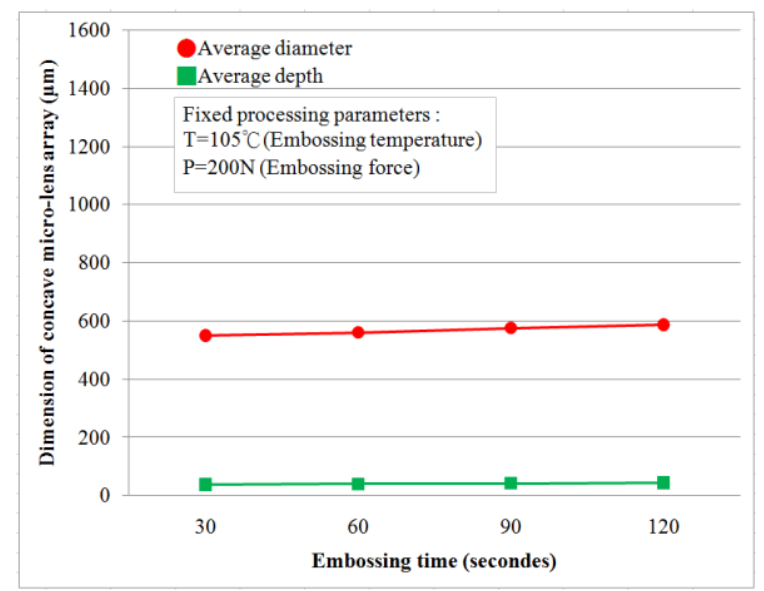

Figure 11 Effect of embossing time on the dimension of concave lens arrays

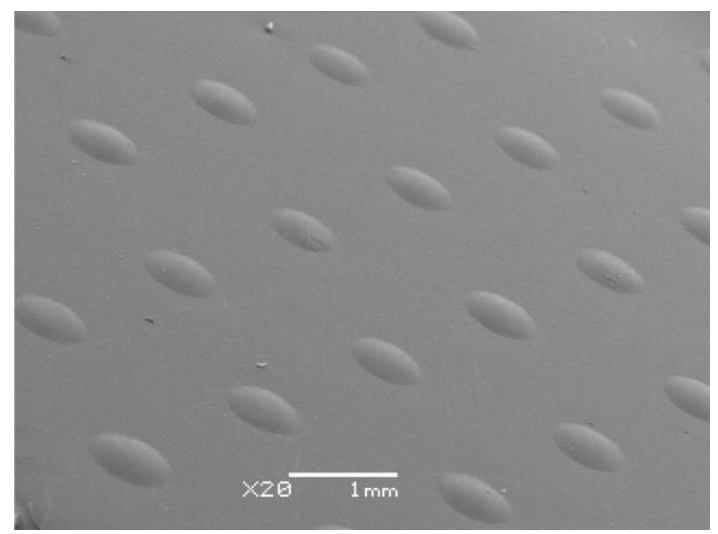

(a) Replicated convex lens array with diameter of $550 \mu \mathrm{m}$

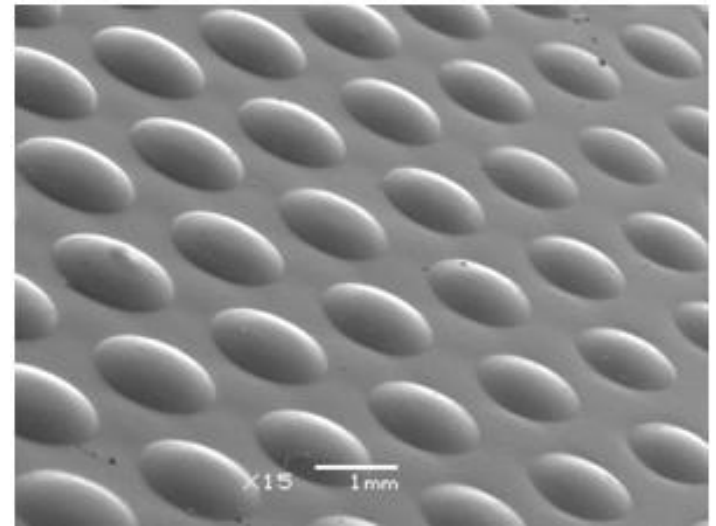

(b) Replicated convex lens array with diameter of $1512 \mu \mathrm{m}$ Figure 12 Replicated convex lens arrays with different diameter 


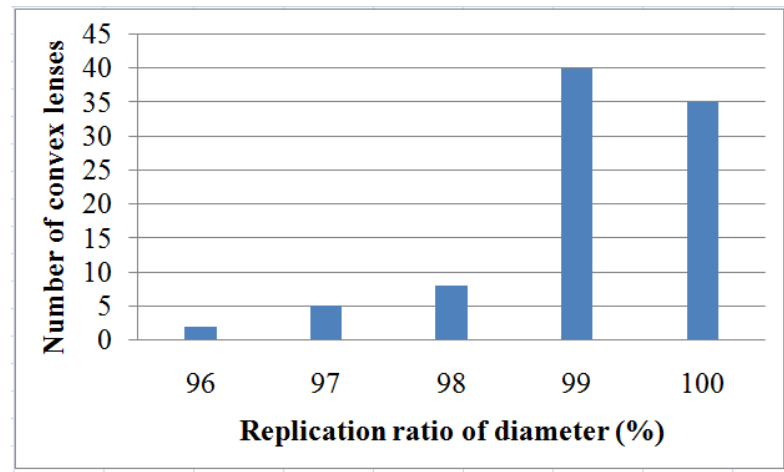

Figure 13 The replication ratio of diameter of a convex lens array

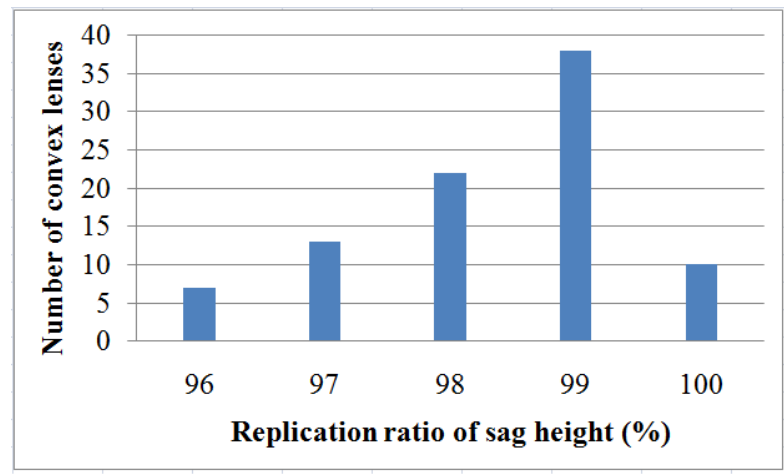

Figure 14 The replication ratio of sag height of a convex lens array

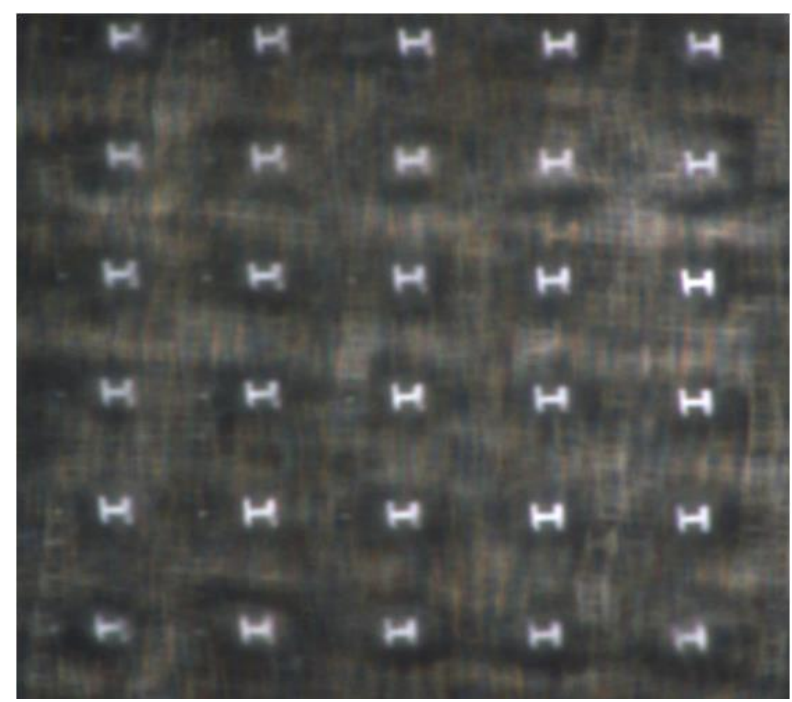

Figure 15 The imaging result of the convex lens array

\section{CONCLUSION}

In this paper, a rapid manufacturing process for the production of concave and convex lens arrays using the hot embossing process with a polymer substrate and a small steel ball array was demonstrated. The experimental result suggests that the diameter and depth of the concave lens arrays could be changed and controlled by adjusting the processing conditions of the hot embossing process. After undergoing a vacuumassisted UV-molding process with the concave lens array, a polymer convex lens array device could be successfully replicated. The replication ratios of diameter and sag height of the UV-molded convex lens arrays from the molds were higher than $98 \%$. The measured optical properties and surface quality of the concave and convex lens arrays were uniform and good. These experimental results show that the proposed method is ideal for mass production of concave and convex lens arrays.

\section{ACKNOWLEDGMENTS}

This study was supported by the Ministry of Science and Technology of Taiwan, R.O.C. (MOST 106-2221-E151-018). The experimental and technical support from the Center for Micro/Nano Science and Technology (NCKU) are gratefully acknowledged.

\section{REFERENCES}

[1] Lin, C.P., Yang, H., and Chao, C.K. 2003. Hexagonal microlens array modeling and fabrication using a thermal reflow process. J. Micromech. Microeng. 13, 775-781.

[2] Naessens, K, Ottevaere, H, Baets, R, Daele, P.V., and Thienpont H. 2004. Direct writing of microlenses in polycarbonate with excimer laser ablation. Appl. Opt. 42, 6349-6359.

[3] Ishii, Y., Koike, S., Arai, Y. and Ando, Y. 2000. Inkjet fabrication of polymer microlens for I/O chip packaging. Jpn. J. Appl. Phys. 39, 1490-1493.

[4] Lee, S.K., Lee, K.C. and Lee, S.S. 2005. A simple method for microlens fabrication by the modified LIGA process. J. Micromech. Microeng. 12, 334340.

[5] Totsu, K. and Esashi, M. 2005. Gray-scale photolithography using maskless exposure system. J. Vac. Sci. Technol. B 23(4), 1487-1490.

[6] Kim, S.M., and Kang, S. 2003. Replication qualities and optical properties of UV-moulded microlens arrays. J. Phys. D: Appl. Phys. 36, 2451-2456.

[7] Kunnavakkam, M.V. Houlihan, F.M., Schlax, M., Liddle, J.A., Kolodner, P., Nalamasu, O., and Rogers, J.A. 2003. Low-cost, low-loss microlens arrays fabricated by soft-lithography replication process. Appl. Phys. Lett. 82, 1152-1154.

[8] Lee, B.K., Kim, D.S., and Kwon, T.H. 2004. Replication of microlens arrays by injection molding. Microsyst. Technol. 10, 531-535.

[9] Hocheng, H., Wen, T.T., and Yang, S.Y. 2008. Replication of microlens arrays by gas-assisted hot embossing. Materials and Manufacturing Processes 23, 261-268. 
[10]Lin, T.H., Yang, H., and Chao, C.K. 2007, Concave microlens array mold fabrication in photoresist using UV proximity printing. Microsystem Technologies 13, 1537-1543.

[11]Chang, S., and Yoon, J.B. 2004. Shape-controlled, high fill-factor microlens arrays fabricated by a 3D diffuser lithography and plastic replication method. Optics Express 12, 6366-6371.

[12] Li, F., Chen, S., Luo, H., Zhou, Y., Lai, J., and Gao, Y. 2012. Fabrication and characterization of polydimethylsiloxane concave microlens array. Optics \& Laser Technology 44, 1054-1059.
[13] Chen, F., Liu, H., Yang, Q., Wang, X., Hou, C., Bian, H., Liang, W., Si, J., and Hou, X. 2010. Maskless fabrication of concave microlens arrays on silica glasses by a femtosecond-laser-enhanced local wet etching method. Optics Express 18, 2033420343.

[14] Zhang, P., Londe, G., Sung, J., Johnson, E., Lee, M., and Cho, H.J. 2007. Microlens fabrication using an etched glass master. Microsystem Technologies $13,339-342$. 